\title{
X45 - Biological Sciences Tutorial: Tips and Tricks for High-Pressure Freezing / Freeze Substitution.
}

\author{
Martin Schauflinger ${ }^{1 *}$ \\ 1. Electron Microscopy Facility, University of Missouri, Columbia, Missouri \\ * Corresponding author: schauflingerm@missouri.edu
}

Cryofixation by high pressure freezing followed by freeze substitution (HPF/FS) results in superior preservation of biological ultrastructure when compared to conventional protocols, in which the samples are submerged in buffered fixative solution and dehydrated through a graded series of alcohol. The advantages of cryofixation are based on the much faster rate of fixation, which results in near simultaneous stabilization of all cellular components [1]-[3]. Freeze substitution is superior over conventional chemical fixation and dehydration due to the relative gentle nature of low temperature processing, which results in less artifacts and better preservation of cellular ultrastructure and antigenicity [4], [5]. The application of HPF/FS has gained significant interest in the EM community in recent decades because it combines the superior morphology preservation of HPF with the ability to stably embed the sample into resin for subsequent applications like thin sectioning for transmission electron microscopy/tomography, or serial block face imaging.

Freezing samples with fast cooling rates and under high pressure allows to increase the depth of good freezing to several hundred microns, which is not achievable with other cyrofixation methods like plunge freezing. With the introduction of commercial HPF instruments it became possible to routinely freeze biological samples successfully, that is with a minimum of destructive ice crystal formation. One of the most significant restrictions of HPF is the sample size. Theoretically samples of up to $0.6 \mathrm{~mm}$ thickness can be frozen well [1], however in practice this is hardly achieved, and thus producing sufficiently thin samples is a particular problem. Probably the most challenging and important step for HPF is sample loading into specimen carriers. There is a multitude of available specimen carriers and filler materials to surround the sample during the freezing process.

Freeze substitution is the process in which water frozen within cells is replaced by a solvent at temperatures well below the freezing point of water. By addition of chemical fixatives to the FS solution, the HPF/FS samples are dehydrated, fixed and stained at low temperature. Unlike HPF, the FS process does not necessarily require expensive instrumentation. Traditional FS protocols last several days, however, quick freeze substitution in the order of a couple hours works in many occasions and considerably speeds up the lengthy process [7]. A combination of fixatives and staining agents can be employed in a FS cocktail, and sometimes it is necessary to include several subsequent incubations to achieve the desired staining. While osmium tetroxide and uranyl acetate are the most commonly used heavy metal stains, I want to discuss some lesser known chemicals that can be used to increase staining contrast. Loading samples up with lots of heavy metal stain is especially helpful when thin sectioning and post staining is not possible, for instance in tomography or block face imaging techniques.

In this biological sciences tutorial, I will elaborate on some of the methodical intricacies and share tips and tricks to make it easier to successfully prepare samples by the HPF/FS technique. There are many factors that can be changed to influence the outcome of an HPF/FS experiment, and much helpful literature is available addressing the practical nature of HPF/FS [6]. While many publications rightfully 
praise the benefits of HPF/FS it is important to understand and recognize artefacts that can occur during this process. I will discuss how to detect commonly observed artefacts such as freeze damage caused by formation of large water ice crystals, as well as other artefacts induced by high pressure. We will briefly discuss the theoretical basis of the HPF/FS process, but more importantly for this format, the best current practices that are applied for biological specimens today.

\section{References:}

[1] R Dahl and LA Staehelin, Journal of Electron Microscopy Technique 13 (1989) p. 165.

[2] J Dubochet in "Cellular Electron Microscopy", ed. JR McIntosh, (Elsevier) p. 7.

[3] H Moor in "Cryotechniques in Biological Electron Microscopy", eds. RA Steinbrecht and K Zierold (Springer, Berlin, Heidelberg) p. 175.

[4] S Hippe-Sanwald, Microscopy Research and Technique 24 (1993) p. 400.

[5] R Shiurba, International Review of Cytology 206 (2001) p. 45.

[6] KL McDonald et al in "Electron Microscopy of Model Systems”, ed. T Müller-Reichert (Elsevier) p. 671.

[7] KL McDonald, Protoplasma 251 (2014) p. 429.
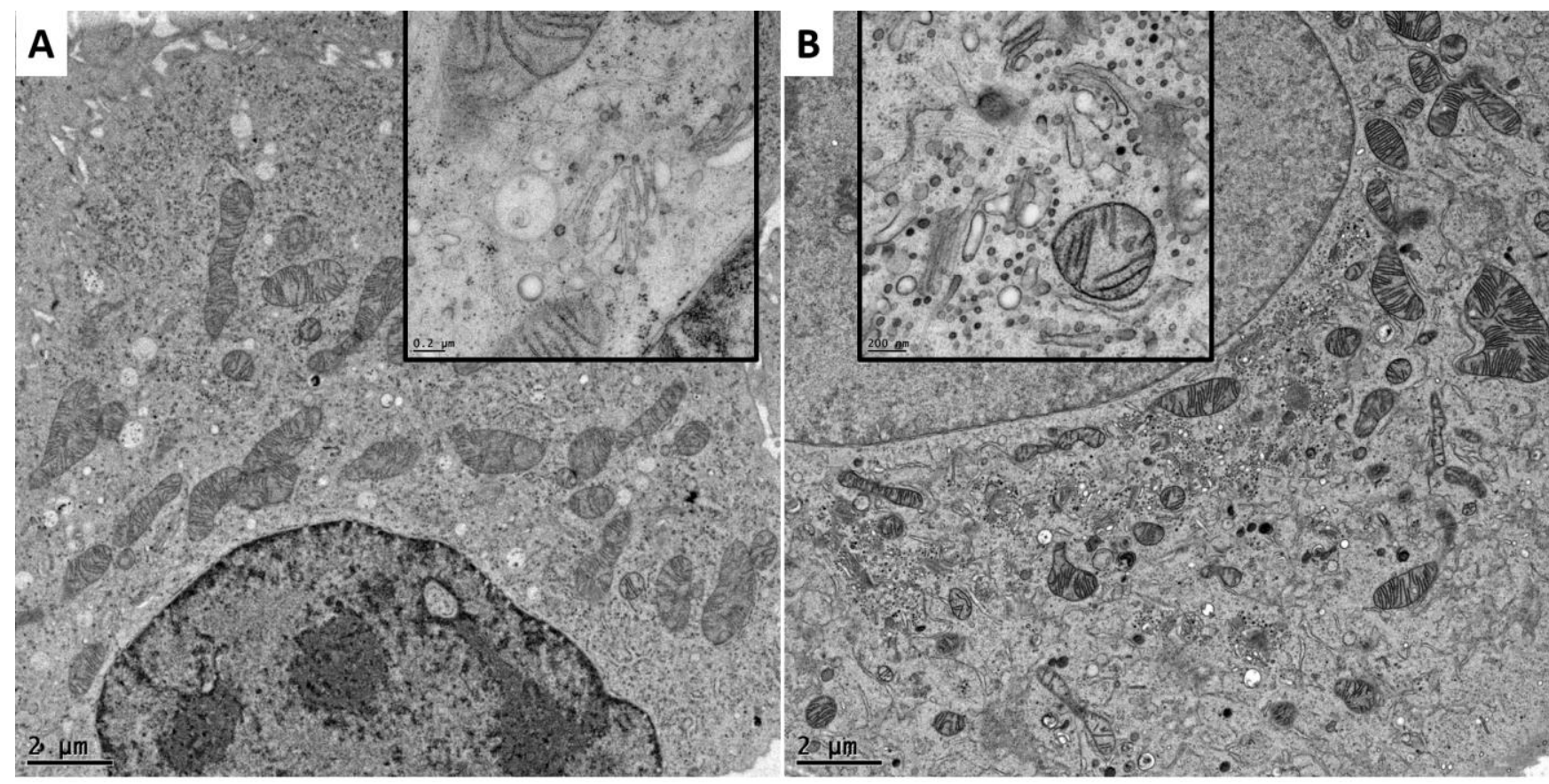

Figure 1. High pressure frozen cultured mammalian cells were freeze substituted in less than 2 hours in a mix of osmium tetroxide and uranyl acetate without (A) and with (B) addition of imidazole which is one of many reagents that can be used to increase membrane contrast. 